\title{
Anisotrope Kernresonanzfluoreszenzausbeute von Cr-Atomen in einem Vanadiumeinkristall
}

\author{
J. Kalus, J. Neuhauser und E. Pückert \\ Physik-Department der Technischen Hochschule München \\ (Z. Naturforschg. 22 a, 788-792 [1967]; eingegangen am 27. Januar 1967)
}

\begin{abstract}
Die bereits früher für einige Kristallrichtungen nachgewiesene Anisotropie der Kernresonanzfluoreszenz ${ }^{1}$ am 1,43 MeV-Niveau von $\mathrm{Cr}^{52}$ an neutronenbestrahlten $\mathrm{V}^{51}$-Einkristallen, die infolge der anisotropen Abbremsung der Cr-Atome (Anfangsenergie 20-90 eV) auftritt, wurde bei Drehung des Kristalles um die $\langle 110\rangle$-Achse in $10^{\circ}$-Intervallen gemessen. Der Anisotropieeffekt beträgt bis zu 10\%. Es zeigt sich, daß ein Hartkugelmodell mit sprungartiger Abbremsung nach dem ersten Stoß mit einem Nachbaratom zur Deutung der Messungen nicht ausreicht. Dagegen liefert ein realistischeres Modell mit Mehr-Stufen-Abbremsung gute Übereinstimmung mit den Messungen.
\end{abstract}

\section{Grundlagen}

Die $\beta-\gamma$-Kaskade beim Zerfall von $\mathrm{V}^{52}$ (Abb. 1) ermöglicht Kernresonanzfluoreszenz (KRF)-Messungen, die Aussagen über die Abbremsung der nach dem $\beta$-Zerfall entstehenden Cr-Atome erlauben ${ }^{2,3}$.

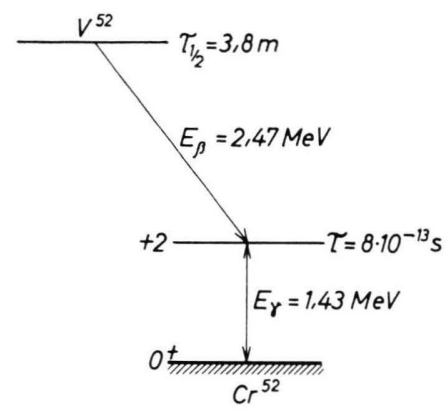

Abb. 1. Zerfallsschema von $\mathrm{V}^{52}$.

Im allgemeinen tritt bei Emission bzw. Absorption von $\gamma$-Quanten je ein Rückstoßenergieverlust von

$$
\Delta E=E_{\gamma}^{2} /\left(2 M c^{2}\right)
$$

auf $\left(E_{\gamma}=\right.$ Energie der $\gamma$-Quanten, $M=$ Kernmasse, $c=$ Lichtgeschwindigkeit), so daß keine Überlappung zwischen Emissions- und Absorptionslinie eintritt, da

$$
\Delta E \gg \sqrt{\Gamma^{2}+\Delta^{2}} .
$$

$\Gamma$ ist die natürliche Linienbreite des angeregten Kernniveaus und $\Delta$ die Doppler-Verbreiterung der Linie, hervorgerufen durch die Temperaturbewegung der Kerne.

1 F. Jähnig u. J. Kalus, Z. Naturforschg. 20 a, 387 [1965].

2 J. B. Cumming, A. Schwarzschild, A. W. Sunyar u. N. T. Porile, Phys. Rev. 120, 2128 [1960].
Infolge der extremen Doppler-Verbreiterung der Emissionslinie durch den $\beta$-Kernrückstoß wird für einen Teil der Quanten der Energieverlust $2 \Delta E$ ausgeglichen, so daß in diesem Fall KRF möglich wird. Dies ist der Fall, wenn

$$
v_{z}=E_{\gamma} / M c
$$

ist. $v_{z}$ ist die Komponente der Rückstoßgeschwindigkeit in Richtung zum Resonanzstreuer.

Die KRF-Ausbeute ist proportional $\mathrm{zu}^{4}$

$$
A=\int N(E) \sigma(E) \mathrm{d} E .
$$

Da $N(E)$, die spektrale Verteilung der auf den Streuer auftreffenden Quanten, eine im Vergleich zum Wirkungsquerschnitt $\sigma(E)$ für KRF langsam veränderliche Funktion ist, kann man schreiben:

$$
A=N\left(E_{\mathrm{r}}\right) \int \sigma(E) \mathrm{d} E=\text { const } N\left(E_{\mathrm{r}}\right) .
$$

$\mathrm{N}\left(\mathrm{E}_{\mathrm{r}}\right)$ ist wegen der Resonanzbedingung (3) abhängig vom Rückstoßimpuls $p$ zur Zeit $t$ der $\gamma$-Emission und damit von der Abbremsung der Chromrückstoßatome im Kristall. Das führt zu einer Richtungsabhängigkeit der KRF-Ausbeute an Einkristallen ${ }^{\mathbf{1}}$, welche die Symmetrie des Gitters wiedergibt.

\section{Experiment}

Die KRF-Messung wurde als Streuexperiment durchgeführt. Durch die Wahl eines großen Streuwinkels lassen sich die Compton-gestreuten Quanten im Detektor gut von den resonant gestreuten Quanten trennen. Die Anordnung von Streuer, Quelle, Detektor und Monitor ist aus Abb.2 zu ersehen. Die pfennigförmigen $\mathrm{V}^{51}$-Einkristallscheibchen wurden beiderseits etwa $3 \mathrm{~mm}$

${ }^{3}$ J. Kalus, Z. Naturforschg. 20 a, 391 [1965].

4 F. R. Metzger, Progr. Nucl. Phys. 7, 53 [1959]. 
hoch mit Eisenpulver (mit Uhu-plus als Bindemittel vermischt) beschichtet. Da die Selbstabsorption von Einkristall und Eisenpulver ungefähr gleich groß ist, ergibt sich bei nicht exakt horizontaler Lage der Proben in erster Näherung keine Intensitätsänderung. Vanadium besitzt ein kubisch raumzentriertes Gitter. Die Symmetrieachse der Einkristallscheibchen fiel mit der $\langle 110\rangle$-Richtung zusammen. Es wurden die relativen Resonanzausbeu:en für 10 verschiedene Kristallorientierungen bestimmt, indem die Quelle um diese $\langle 110\rangle$. Achse in Schritten von je $10^{\circ}$ gedreht wurde.

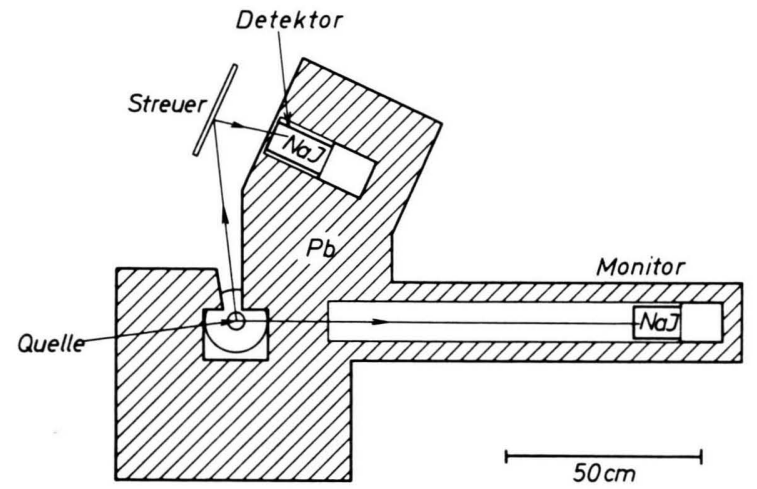

Abb. 2. Versuchsaufbau (Schnitt in Höhe der Quelle).

Das radioaktive $\mathrm{V}^{52}$ wurde durch Neutronenbestrahlung der $\mathrm{V}^{51}$-Einkristalle am Forschungsreaktor München erhalten. Bei einer Bestrahlungszeit von 8 Minuten betrug die Probenstärke etwa 3 Curie. Es wurden 14 annähernd gleiche Einkristalle verwendet, deren etwas unterschiedliche Aktivität mittels eines Monitors auf einen durchschnittlichen Wert normiert wurde. Wegen der kleinen Halbwertszeit von 3,8 Minuten wurde die aktive Probe, in ein Polyäthylen-Rabbit verpackt, über eine Rohrpostleitung vom Reaktorkern direkt in die Meßapparatur geschossen. Dort wurde das Rabbit in einem auf Kugellager drehbaren Messingrohr aufgefangen, in ihm zentriert und mit dem Rohr in die gewünschte Lage gedreht. Der Streuer bestand aus Chrompulver, das mit Araldit als Bindemittel zu einer festen Platte von $25 \mathrm{~cm} \times 20 \mathrm{~cm} \times 2 \mathrm{~cm}$ mit einem Flächengewicht von $6 \mathrm{~g} / \mathrm{cm}^{2}$ verarbeitet wurde. Der Vergleichsstreuer war aus Eisen. Das Energiespektrum des $3^{\prime \prime} \times 3^{\prime \prime} \mathrm{NaJ}(\mathrm{Tl})$-Detektors wurde mit einem RidlVielkanalanalysator aufgenommen (Abb. 3). Der $\mathrm{NaJ}(\mathrm{Tl})$-Kristall war durch $10 \mathrm{~mm}$ Blei abgeschirmt, das vorwiegend niederenergetische Compton-gestreute Quanten absorbiert.

Die normierten Ergebnisse der Messungen sind mit Angabe der statistischen Fehler in Abb. 4 a dargestellt.

Wegen der endlichen Ausdehnung des Streuers tragen zur Resonanzausbeute auch $\gamma$-Quanten bei, deren Richtung von der zu untersuchenden um maximal $6^{\circ}$ abweicht, wodurch die Extrema der Kurve 4 a etwas abgeflacht werden (besonders im Bereich von $90^{\circ}$ ).

5 C. Erginsoy, G. H. Vineyard, A. Englert u. A. Shimizu, Phys. Rev. 133 A, 595 [1964] und 139 A, 118 [1965].

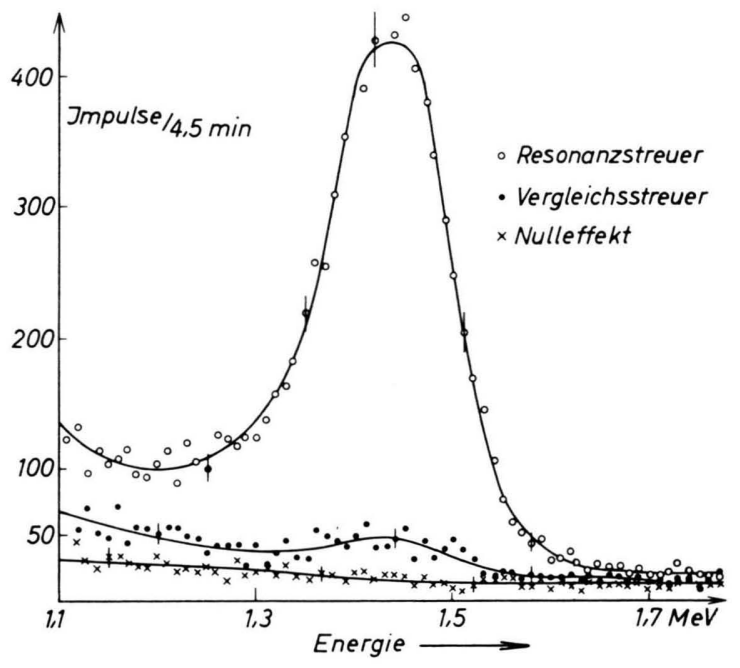

Abb. 3. Beispiel einer Meßkurve. Impulshöhenspektrum des Resonanzeffektes $\mathrm{O}-\mathrm{O}-\mathrm{O}$, des Vergleichsstreuereffektes - - und des Nulleffektes $x-x-x$.

\section{Diskussion}

\section{a) Einfaches Hartkugelmodell}

Für den zeitlichen Verlauf der Geschwindigkeit des Cr-Atoms nehmen wir folgende einfache Abbremsfunktion an:

$$
v_{\mathrm{k}}(t)=\left\{\begin{array}{lll}
v_{0} & \text { für } & 0<t<T, \\
0 & \text { für } & t>T,
\end{array}\right.
$$

wobei $v_{0}$ die Anfangsgeschwindigkeit und $T=s /|v|$ die Flugzeit des Atoms bis zum ersten Stoß mit einem Nachbaratom ist. $s$ ist der freie Flugweg. Wir verwenden ein Hartkugelmodell mit energieunabhängigem Stoßradius, das noch insoweit vereinfacht ist, als das Primäratom nach einem Stoß vollkommen abgebremst wird. Dies ist, falls das Primäratom relativ zentral auf ein nächstes Nachbaratom zufliegt, eine gute Näherung ${ }^{5}$.

Die Wahrscheinlichkeit, daß die Emission des Quants während der Flugzeit $T$ erfolgt, ist

$W_{T}=\frac{1}{\tau} \int_{0}^{T} \exp (-t / \tau) \mathrm{d} t \approx T / \tau=s /(|v| \tau)$,

da $\tau$, die mittlere Lebensdauer des angeregten Kernniveaus $\left(\tau=8 \cdot 10^{-13} \mathrm{sec}\right)$, viel länger als $T$ (ca. $\left.2 \cdot 10^{-14} \mathrm{sec}\right)$ ist.

Die Wahrscheinlichkeit, daß KRF eintritt, kann man als Funktion von $v_{z}$ folgendermaßen schreiben:

$$
W=\operatorname{prop} \int \mathrm{d} \Omega\left(v_{z}\right) \sigma\left(v_{z}\right) W_{T} .
$$


$\mathrm{d} \Omega$ ist das Raumwinkelelement, in das der Rückstoßkern fliegt, $\sigma\left(v_{z}\right)$ ist der Wirkungsquerschnitt für Resonanzstreuung. Da das Rückstoßspektrum beim Zerfall kontinuierlich ist, muß man noch über die Geschwindigkeitsverteilung $H(|v|) \mathrm{d}|v|$ mitteln. Die Gesamtwahrscheinlichkeit für die KRF-Ausbeute wird dann:

$$
W_{\mathrm{KRF}}=\operatorname{prop} \int_{v_{\min }}^{v_{\max }} \frac{\mathrm{d} \Omega\left(v_{z}\right) \sigma\left(v_{z}\right) s H(|v|)}{|v|} \mathrm{d}|v| .
$$

$v_{\text {min }}$ ist die kleinste Geschwindigkeit, die die Resonanzbedingung (3) erfüllt, also

$$
v_{\min }=v_{\text {res }}=E_{\gamma} / M c .
$$

$v_{\max }$ folgt aus dem maximalen Rückstoßimpuls. $v_{\max }$ erfüllt die Resonanzbedingung (3), wenn der Impulsvektor einen Winkel von $\vartheta_{\max }=61^{\circ}$ mit der $z$ Richtung (Richtung Quelle-Streuer) einschließt.

Es können also nur Impulse innerhalb eines Kreiskegels um die $z$-Achse mit einem halben Öffnungswinkel $\vartheta_{\max }$ zur KRF-Ausbeute beitragen. Setzt man in (9) $\sigma\left(v_{z}\right)=\bar{\sigma} \cdot \delta\left(v_{z}-v_{\text {res }}\right)$ und integriert man über $v$, so erhält man

$$
W_{\mathrm{KRF}}=\operatorname{prop} \int_{0}^{61^{\circ}} H\left(v_{\mathrm{res}} / \cos \vartheta\right)\langle s(\vartheta, \varphi)\rangle_{\varphi} \sin \vartheta \mathrm{d} \vartheta .
$$

$\langle s(\vartheta, \varphi)\rangle_{\varphi}$ ist der Mittelwert aller freien Flugwege auf einem Kreiskegel mit dem halben Öffnungswinkel $\vartheta$.

$H(|v|)$ wird numerisch aus dem Spektrum berechnet, wobei versuchsweise die nicht bekannnte Neutrinowinkelkorrelationskonstante ${ }^{6} \lambda$ gleich 0,1 und -1 gesetzt wird. Das Integral (11) wurde auf der Rechenmaschine TR 4 für verschiedene Kristallrichtungen und Ausgangsparameter berechnet. Ein Vergleich der berechneten Werte mit den experimentellen zeigt jedoch keine sinnvolle Übereinstimmung (s. Abb. 4b). Das bedeutet, daß die Abbremsfunktion (6) bei nichtzentralen Stößen mit den nächsten Nachbarn keine gute Näherung ist. Die Abbremsung verläuft in diesen Fällen komplizierter und trägt zur KRF-Ausbeute stark bei. Dies ergibt sich auch aus den Rechnungen von ERginsoy und Mitarbeitern ${ }^{5}$.

\section{b) Einfaches Abbremsmodell}

Eine wesentliche Verbesserung des einfachen Hartkugelmodells erhält man, wenn man die tatsächlichen Potentialverhältnisse, die das Rückstoßatom in der näheren Umgebung vorfindet, folgendermaßen annähert: a) Harte Kugeln an den Gitterplätzen, wie beim vorigen Modell. Der Radius der Kugeln kann energieabhängig gemacht werden, z. B. unter Verwendung eines üblichen Zweiteilchenpotentials ${ }^{5}$ der Form $\varrho=A \cdot e^{-B x}$ ( $x$ ist der Abstand zweier Atome, $A$ und $B$ sind Konstanten). Damit erhält man den wesentlichen Beitrag der Anisotropie.

$\beta$ ) Zwischen den harten Kugeln nähern wir den dort herrschenden relativ flachen Potentialverlauf durch konzentrisch um den Ursprung angeordnete Potentialstufen an.

Dies bewirkt eine starke Änderung der ursprünglichen Geschwindigkeitsverteilung. Solange sich die Primärteilchen innerhalb einer Kugel vom Radius $r=0,4 a \quad(a=$ Gitterkonstante $)$ befinden, erfahren sie keine wesentliche Energieänderung und Ablenkung. Hier gilt das primäre Rückstoßspektrum.

In der Kugelschale $0,4 a<r<0,6 a$ treten die Teilchen in Wechselwirkung mit den nächsten Nachbarn. Ein Teil wird durch direkten Stoß mit den nächsten Nachbarn völlig abgebremst. Die Geschwindigkeitsverteilung der restlichen Teilchen wird durch die Wirkung des flachen Potentialverlaufes zu kleineren Geschwindigkeiten hin verschoben. Eine hohe Schwelle müssen die Teilchen bei etwa $r=0,6 a$ überwinden, wenn sie zwischen den nächsten Nachbarn hindurchfliegen. Dementsprechend wird die Geschwindigkeitsverteilung für $r>0,6 a$ zu wesentlich tieferen Geschwindigkeiten hin verschoben.

In diesem Modell wird (11) in Einzelintegrale zerlegt :

$$
W_{\mathrm{KRF}}=\operatorname{prop} \sum_{\nu=1}^{3} \int_{0}^{61^{\circ}} H_{\nu}\left(v_{\mathrm{res}} / \cos \vartheta\right)\left\langle s_{\nu}\right\rangle_{\varphi} \sin \vartheta \mathrm{d} \vartheta
$$

mit

$$
\begin{aligned}
& s_{1}=\left\{\begin{array}{c}
s \quad \text { für } \quad s<0,4 a, \\
0,4 \text { für } \quad s>0,4 a,
\end{array}\right. \\
& s_{2}=\left\{\begin{array}{cll}
0 & \text { für } & s<0,4 a, \\
s-0,4 & \text { für } & 0,4 a<s<0,6 a, \\
0,2 & \text { für } & s>0,6 a,
\end{array}\right. \\
& s_{3}=\left\{\begin{array}{cll}
0 & \text { für } & s<0,6 a, \\
s-0,6 & \text { für } & s>0,6 a ;
\end{array} s_{1}+s_{2}+s_{3}=s .\right.
\end{aligned}
$$

Bei der Rechnung wurden folgende Geschwindigkeitsverteilungen verwendet:

$H_{1}$ war das ursprüngliche Rückstoßspektrum für $\lambda=0 . H_{2}$ ergibt sich aus $H_{1}$ durch Verschiebung um ca. $15 \mathrm{eV}$ zu niedrigeren Energien. $H_{3}$ wurde versuchsweise als Überlagerung von zwei, dem Ur- 
sprung nach verschiedenen Anteilen von je gleichem Gewicht gewählt $\left(H_{3}=H_{31}+H_{32}\right)$ :

1. $H_{31}$ entsteht durch Verschiebung des ursprünglichen Spektrums $H_{1}$ um $30 \mathrm{eV}$ zu niedrigeren Energien hin.

2. Für $H_{32}$ wurde versuchsweise eine Verteilung gewählt, die man erhält, wenn man die Primärteilchen an freien harten Kugeln stoßen läßt. Vernachlässigt man $H_{32}$, so ist die Übereinstimmung schlecht.

Die Höhe der Potentialschwellen von 15 (30) eV läßt sich aus BorN-Meyer-Potentialen, wie sie ERGINSOY ${ }^{5}$ bei Kupfer verwendet hat, roh abschätzen. Die Aufteilung von $H_{3}$ in die zwei Anteile ist in roher Näherung durch folgende Überlegung gerechtfertigt:

Fliegt ein Atom so zwischen Nachbaratomen hindurch, daß seine Richtung kaum, die Geschwindigkeit dagegen stark geändert wird, so kann das gut durch eine konzentrisch angeordnete Potentialschwelle beschrieben werden $\left(H_{31}\right)$.

Fliegt ein Atom dagegen so, daß in diesem Fall sowohl Richtung wie auch Geschwindigkeit stark geändert werden, so genügt die konzentrisch angeordnete Potentialschwelle nicht mehr zur Beschreibung.

Der Hartkugelradius war $R=0,18 a=$ const. In diesem Modell erfolgt die Abbremsung also stufenweise. Richtungsänderungen werden nicht explizit berechnet, aber qualitativ in $H_{3}$ berücksichtigt.

Mit dieser speziellen Wahl der Modellparameter erhält man bis auf geringe Abweichungen die im Experiment gemessene Kurve (Abb. 4c). Variationen der Potentialschwellenradien und des Hartkugelradius um weniger als $10 \%$ verändern die Ausbeutekurve noch nicht wesentlich. Auch negative oder schwach positive $\lambda$-Werte liefern noch brauchbare Ergebnisse. Dagegen erhält man in diesem Modell für $\lambda \lesssim+1$ ähnlich schlechte Kurven wie in der primitiven Hartkugel-Näherung.

Die V-A-Theorie des $\beta$-Zerfalls ${ }^{6}$ sagt für Übergänge ohne Paritätsänderung und mit $\Delta I=0 \quad \lambda$ Werte zwischen $-\frac{1}{3}$ und +1 voraus. Somit würde die vorliegende Messung einem reinen Fermi-Übergang $(\lambda=+1)$ widersprechen. Diese Aussage gilt jedoch nur unter der Einschränkung, daß das einfache Abbremsmodell die Rückstoßabbremsung hinreichend gut beschreibt ${ }^{7}$.

6 O. Kofoed-Hansen, in Alpha-, Beta- and Gamma-Ray Spectroscopy, Vol. 2, p .1411-1412, edited by KaI Siegbahn, North-Holland Publish. Co., Amsterdam 1965.

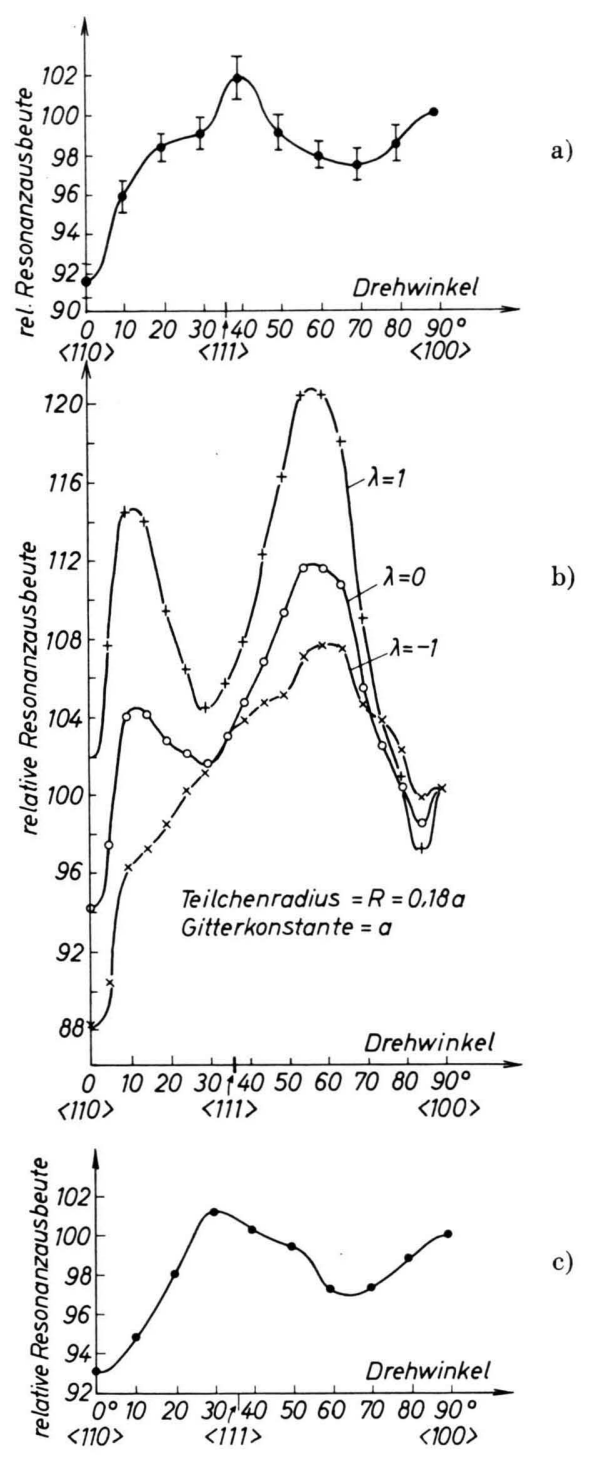

Abb. 4 a. Meßwerte der relativen Resonanzausbeuten für verschiedene Kristallrichtungen.

Abb. 4 b. Relative Resonanzausbeuten für verschiedene Kristallrichtungen, berechnet mit dem „Einfachen Hartkugelmodell“.

Abb. 4c. Relative Resonanzausbeuten für verschiedene Kristallrichtungen, berechnet mit dem „Einfachen Abbremsmodell“.

Es ist ohne Schwierigkeit möglich, die Abbremsung unter Benutzung von realistischen BorN-MEyerPotentialen, wie sie Erginsoy bei seinen Rechnungen an $\alpha$-Eisen verwendet, zu berechnen und die KRFAusbeute mit den genauen Abbremsfunktionen zu

7 Nach neuen Messungen ist $\lambda=-\frac{1}{3}$ : S. D. Bцоoм u. L. G. MAnN, Nucl. Phys. A 93, 252 [1967]. 
berechnen. Da die Anisotropie der KRF-Ausbeute, wie wir gesehen haben, modellabhängig ist, lassen sich die zunächst willkürlich oder näherungsweise angenommenen Konstanten im Potential durch Variation derselben und Vergleich der Rechenergebnisse mit den experimentellen Werten (das gleiche gilt für die Neutrinowinkelkorrelationskonstante $\lambda$ ) bestimmen. Wir haben dieses Verfahren wegen der dafür notwendigen langen Rechenzeit nicht durchführen können.

Herrn Prof. Maier-Leibnitz danken wir für die Unterstützung bei der Arbeit. Unser Dank gilt auch der Leitung und der Bestrahlungsgruppe des Forschungsreaktors München.

\title{
Untersuchung von (n, $\gamma)$-Rückstoßschäden in Vanadium mit der Kernresonanzfluoreszenzmethode
}

\author{
J. Kalus und J. Neuhauser \\ Physik-Department der Technischen Hochschule München \\ (Z. Naturforschg. 22 a, 792-796 [1967] ; eingegangen am 27. Januar 1967)
}

\begin{abstract}
Es wurden Kernresonanzfluoreszenzmessungen am 1,43 MeV-Niveau von $\mathrm{Cr}^{52}$ bei $10^{\circ}$ und $300{ }^{\circ} \mathrm{K}$ durchgeführt. Neutronenbestrahltes polykristallines Vanadiummetall und ein Vanadiumeinkristall dienten als $\gamma$-Strahlenquelle für das Resonanzstreuexperiment. Mit Hilfe eines einfachen Abbremsmodells erhält man Aussagen über die Konzentration von Gitterdefekten in der nächsten Umgebung des $\mathrm{Cr}^{52}$-Atoms bei $10^{\circ} \mathrm{K}$. Unter Vernachlässigung von Doppelfehlstellen erhält man :

1. Konzentration von Normalgitterplätzen: $\quad 0<c_{\text {NG }}<0,92 \pm 0,08$,

2. Konzentration von Zwischengitterplätzen:

$0<c_{\mathrm{ZG}}<0,36 \pm 0,03$

3. Konzentration von Leerstellen an nächster Nachbarstelle: $\quad 0<c_{\mathrm{L} 1}<0,64 \pm 0,03$,

4. Konzentration von Leerstellen an übernächster Nachbarstelle: $0<c_{\mathrm{L} 2}<0,73 \pm 0,04$.

Die Konzentrationen sind nicht unabhängig voneinander, da $c_{\mathrm{NG}}+c_{\mathrm{ZG}}+c_{\mathrm{L} 1}+c_{\mathrm{L} 2}=1$ ist.
\end{abstract}

\section{Grundlagen}

Die bei der anisotropen Kernresonanzfluoreszenzmessung (KRF) an ${ }^{1,2} V^{51}$ gewonnenen Ergebnisse kann man verwenden, um in einem modifizierten Experiment Aussagen über Punktdefekte in der Umgebung des Primärteilchens zu erhalten. Bei der Bestrahlung im Reaktor mit thermischen Neutronen $\left(\mathrm{V}^{51}(\mathrm{n}, \gamma) \mathrm{V}^{52}\right.$-Prozeß) erhält das Primärteilchen durch den $\gamma$-Rückstoß eine Anfangsenergie von ca. $300 \mathrm{eV}$, die ausreicht, um eine Defektkaskade zu erzeugen. Zur KRF-Messung wird dann die beim nachfolgenden Zerfall von $\mathrm{V}^{52}$ zu Cr${ }^{52}$ emittierte $\beta-\gamma$-Kaskade herangezogen $(\mathrm{Abb} . \mathrm{l})$. Im allgemeinen überlappen

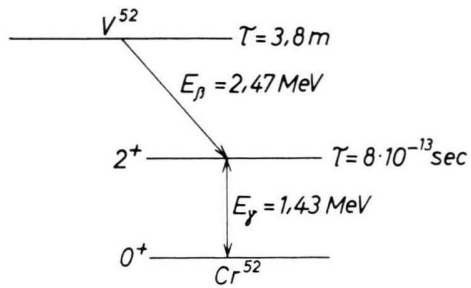

Abb. 1. Zerfallsschema von $\mathrm{V}^{52}$.

1 F. Jähnig u. J. Kalus, Z. Naturforschg. 20 a, 387 [1965]. sich $\gamma$-Emissions- und Absorptionslinie wegen des zweifachen Rückstoßenergieverlustes

$$
2 \Delta E=E_{\gamma}{ }^{2} / M c^{2}
$$

und der im Vergleich dazu geringen Linienbreite nicht $(M=$ Atommasse und $c=$ Lichtgeschwindigkeit).

Der dem $\gamma$-Zerfall vorausgehende $\beta$-Zerfall bewirkt eine Verbreiterung der Emissionslinie, da der $\gamma$-Zerfall vom bewegten Kern aus erfolgt. Das Überlappungsintegral

$$
A=\int N(E) \sigma(E) \mathrm{d} E \approx N\left(E_{\mathrm{R}}\right) \int \sigma(E) \mathrm{d} E
$$

wird dadurch groß und meßbar. $[N(E)=$ spektrale Energieverteilung der $\gamma$-Quanten, $\sigma(E)=$ Wirkungsquerschnitt für KRF-Streuung.] KRF tritt nur dann ein, wenn die Geschwindigkeitskomponente $v_{z}$ des Kerns in Streuerrichtung zur Zeit $t$ der $\gamma$-Emission den Wert

$$
v_{z}=E / M c
$$

besitzt. $A$ wird dadurch eine Funktion der Abbremsung der Cr-Atome an den umgebenden Atomen. Da

\footnotetext{
2 J. Kalus, J. Neuhauser u. E. Pückert, Z. Naturforschg. 22 a, 788 [1967]; voranstehende Arbeit.
} 